\title{
A proteomic approach to the identification of lung cancer markers
}

\author{
Samir Hanash ${ }^{\mathrm{a}, *}$, Franck Brichory ${ }^{\mathrm{a}}$ and \\ David Beer ${ }^{\mathrm{b}}$

 \\ Medical Center, Ann Arbor, MI 48109, USA \\ ${ }^{\mathrm{b}}$ Department of Surgery, University of Michigan \\ Medical Center, Ann Arbor, MI 48109, USA
}

\begin{abstract}
We have developed a comprehensive approach to the identification of protein markers in lung cancer that includes profiling of tumor tissue and cell lines as well as the analysis of serum for autoantibodies to lung tumor antigens. A large number of proteins that are differentially expressed in the major subtypes of lung cancer have been identified by mass spectrometry. A database of protein expression in lung cancer and other types of cancer has been constructed that integrates two-dimensional gel profiles, mass spectrometry data, quantitative protein data and gene expression data at the RNA level, that serves as a resource for biomarker identification. Analysis of the serological response in lung cancer has led to the identification of novel markers detectable in serum of lung cancer patients at the time of diagnosis. The proteomic approach is likely to yield novel classification schemes and novel markers for early diagnosis of lung cancer.
\end{abstract}

Keywords: Lung, cancer, proteomics, markers

\section{Introduction}

We have implemented a comprehensive strategy for the molecular analysis of lung cancer that includes proteomic analysis of lung tumor tissue and cell lines, as well as serum analysis for the identification of lung tumor proteins that induce a serological response in the form of autoantibodies. Although we have relied to date primarily on two-dimensional (2-D) polyacry-

\footnotetext{
* Address for correspondence: Samir Hanash, University of Michigan Medical Center, 1150 W. Medical Center Drive, A520 Medical Science Research Building I, Ann Arbor, MI 48109-0656, USA. Tel.: +1 734763 9311; Fax: +1 734647 8148; E-mail: shanash@ umich.edu.
}

lamide gels for protein separations, the 2-D gel approach is being increasingly complemented with additional analyses using liquid based protein separations and protein microarrays. Proteomic analysis of tissues and cell populations uniquely contributes an understanding of protein post-translational modifications and of the distribution of protein gene products in subcellular compartments. An important objective of our lung cancer effort is the identification of novel markers for early detection.

A large number of studies involving lung cancer have been independently performed in the laboratory. At the protein level, these studies have resulted in over 1000 samples related to lung cancer, that have been processed using 2-D gels and for which information has been recorded in the Lung Protein Database. This number represents a fraction of over 30,000 2-D Gels produced by our group for different studies, including studies of other cancer types. While lung adenocarcinomas represent a major portion of the lung cancer database, other lung tumor types including squamous cell carcinomas and small cell lung cancers are represented, as are control lung tissues. Other 2-D patterns were produced from studies of cell lines that have been manipulated by transfection or by treatment with specific agents, as well as patterns produced after different cell fractionation schemes.

Mass Spectrometry and/or N-terminal sequencing of protein spots from 2-D gels of lung tumor samples or cell lines has led to the identification of a large number of proteins expressed in lung cancer. Also, most identifications made for proteins from a sample type can often be confidently transferred to matching protein spots on master images from lung studies. Figure 1 exhibits some of the progress we have made in identifying proteins in 2-D gels of lung samples.

An important first step in mining lung cancer proteomic data for various applications is to determine the ability of proteomic profiling to distinguish between known types of lung cancer. Specific protein differences between different types of cancer have been identified by other groups. In a recent study of breast, ovary 
and lung tumors, 20 differentially expressed proteins were identified [1] and in a prior study, 16 polypeptides were found to be associated with different histopathological features of lung cancer [2,3]. In a study of 25 adenocarcinomas of the lung, 12 small cell lung cancers, and 16 squamous cell tumors, by our group (manuscript submitted) an initial analysis of protein 2-D patterns uncovered a group of 52 protein spots that differed in average integrated intensity between the three groups in a statistically significant manner. We have identified 39 of this set of 52 spots by either Nterminal sequencing and/or mass spectrometry of spot digests.

\section{Serological approaches for the identification of lung cancer markers}

There is increasing evidence for an immune response to cancer in humans, demonstrated in part by the identification of autoantibodies against a number of intracellular and surface antigens detectable in sera from patients with different cancer types [4-7]. The majority of tumor derived antigens that have been identified as eliciting a humoral response in lung cancer, as in other tumor types, are not the products of mutated genes. They include differentiation antigens and other proteins that are overexpressed in tumors [8]. The oncogenic proteins L-Myc and C-Myc have been found to elicit autoantibodies in a small percentage of patients $[5,9]$. There is some evidence that occurrence of autoantibodies in lung cancer is of prognostic relevance [10-15]. Remarkably, tumor regression has been demonstrated in some patients with small-cell lung carcinoma and autoantibodies to onconeural antigens [15,16]. It is not clear why only a subset of patients with a tumor type develop a humoral response to a particular antigen. Immunogenicity may depend on the level of expression, post-translational modification or other types of processing of a protein, the extent of which may be variable among tumors of a similar type. Other factors that influence the immune response may include variability among individuals and tumors in major histocompatibility complex molecules. Cytokines are also known to affect the immune response and may vary in concentration between tumors or in circulation [17-19].

The identification of panels of tumor antigens that elicit an antibody response may have utility in cancer screening, diagnosis or in establishing prognosis. Such antigens may also have utility in immunotherapy against the disease. There are several approaches for the detection of tumor antigens that induce an immune response. A number of antigens have been detected by screening expression libraries with patient sera [4-6, 20-22]. The merits of our proteomic approach is that it allows proteins, in their modification states as they occur in cells, to be analyzed for their antigenicity. Given that proteins are subject to post-translational modifications, antibodies to epitopes that result from such posttranslational modifications can be detected. Additionally, the 2-D approach allows for serial serum samples to be analyzed much more readily than the screening of expression libraries.

We have implemented a proteomic approach for the identification of tumor antigens that elicit a humoral response [23-25]. To this end, we have utilized 2-D PAGE to simultaneously separate several thousand individual cellular proteins from tumor tissue or tumor cell lines. Separated proteins are transferred onto membranes. Sera from cancer patients are screened individually, for antibodies that react against separated proteins, by Western blot analysis. Proteins that specifically react with sera from cancer patients are identified by mass spectrometric analysis and/or amino acid sequencing and further evaluated with respect to their specificity.

\section{A combined serological and proteomic-based approach to the identification of novel lung cancer markers}

We have identified using our proteomic approach a battery of proteins that induce autoantibodies that are specific for different types of cancer including some that show specificity for lung cancer. The availability of a database of protein expression in lung cancer has facilitated the identification of proteins that induce autoantibodies, in addition to providing valuable information regarding the expression pattern of such protein antigens in different tumor types and cell lines. One such antigen we have identified in lung cancer is protein PGP 9.5 (Fig. 2) [24]. PGP 9.5 was identified as a protein in lung cancer that induces autoantibodies as part of a study in which sera from 64 newly diagnosed patients with lung cancer, from 99 patients with other types of cancer and from 71 non-cancer controls were analyzed for antibody-based reactivity against lung adenocarcinoma proteins resolved by 2-D PAGE. Gels containing separated proteins were blotted and subsequently hybridized with individual sera from patients or controls. Unlike controls, autoantibodies against a protein iden- 




Fig. 1. A small cell lung cancer master image with identified proteins.

tified by mass spectrometry as protein gene product 9.5 (PGP 9.5) were detected in sera from 9 of 64 patients with lung cancer.

Circulating PGP 9.5 antigen was detected in sera from two additional patients with lung cancer, without detectable PGP 9.5 autoantibodies. PGP 9.5 was first identified as a specific marker for neurons and neuroendocrine cells [26]. PGP 9.5 belongs to a family of ubiquitin C-terminal hydrolase (UCH) isoenzymes that play a regulatory role in the ubiquitin system [27].
It has been implicated in the mechanism to remove ubiquitin from ubiquitinated proteins and thus preventing their degradation by proteasomes [28]. Ubiquitination of cellular proteins and their targeting for subsequent degradation via ubiquitin-mediated proteolysis is an important mechanism that regulates the activity of a variety of genes, notably cell cycle genes [27,29]. In lung tumors, increased de-ubiquitination of cyclins by PGP 9.5 may contribute to uncontrolled proliferation [28]. In our study we demonstrated by 2-D PAGE 


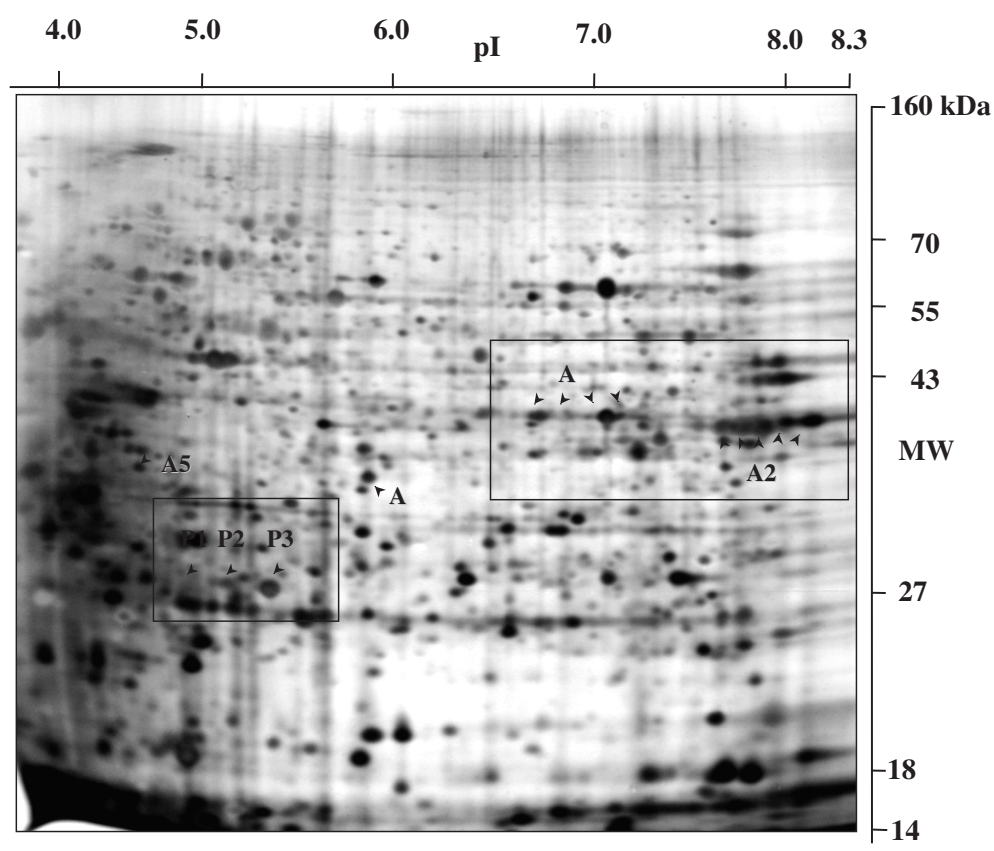

Fig. 2. A 2-D image of a lung adenocarcinoma. Boxed areas containing annexins I and II and PGP 9.5 proteins are presented in Fig. 3.

and Western blot analyses that $80 \%$ of the lung tumors we have studied contained detectable levels of PGP 9.5. In previous analyses by immunohistochemistry, PGP 9.5 was detected in 40-82.5\% of NSCLC and 50-90\% of SCLC [30-33]. Hibi et al reported ectopic expression of PGP 9.5 in lung cancers by SAGE analysis and by immunochemistry $[31,34]$. In primary NSCLCs, $54 \%$ of the cases had positive PGP 9.5 staining, and protein expression was associated with pathological stage (44\% of stage I and $75 \%$ of stages II and IIIA). PGP 9.5 was observed in both SCLC and NSCLC cell lines, independent of neuronal differentiation. Using A549 lung adenocarcinoma cell line, we have demonstrated that PGP 9.5 was present at the cell surface, as well as secreted. Thus, the findings of PGP 9.5 antigen and/or antibodies in serum of patients with lung cancer suggest that PGP 9.5 may have utility in lung cancer screening and diagnosis, as part of a panel of such proteins or their corresponding antibodies, which we have identified.

In another study, sera from 54 newly diagnosed patients with lung cancer and 60 patients with other cancers and from 61 non-cancer controls were analyzed for autoantibodies to lung tumor proteins. Sera from $60 \%$ of patients with lung adenocarcinoma, and $33 \%$ of patients with squamous cell lung carcinoma but none of the non-cancer controls exhibited IgG based reactivity against proteins identified as glycosylated annexins I and/or II. Immunohistochemical analysis showed that annexin I was diffusely expressed in neoplastic cells in lung tumor tissues, whereas annexin II was predominant at the cell surface.

Annexin I is a $37 \mathrm{kDa}$ protein which has been implicated in glucocorticoid induced inhibition of cell growth [35,36]. Annexin II is a $36 \mathrm{kDa}$ protein that occurs in a monomeric form or as a tetramer, associated with the annexin II light chain (p11), which is a member of the S100 family [37,38]. Annexin II has been implicated in cell-cell adhesion and in plasminogen activation and may function as a cell surface receptor [39]. Annexin II tetramers have been shown to interact with procathepsin B on the surface of tumor cells and may be involved in extracellular proteolysis, facilitating tumor invasion and metastasis [40]. Interestingly, annexin I is a target of autoantibodies in autoimmune diseases such as systemic lupus erythematosus [41,42] and rheumatoid arthritis [43]. Annexin II, specifically, has not been previously implicated as a target of autoantibodies in any disorders.

Annexins are known to undergo post-translational modification including glycosylation [44]. Annexin I and annexin II are both phosphorylated by various kinases [45]. In our study, immunoreactivity against annexin I was found to be dependent on $\mathrm{N}$-glycosylation. A potential N-linked glycosylation site is present at positions 42 and 61 from the N-terminus of annexins I and 
A



B

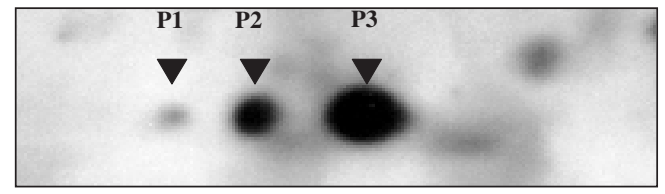

C

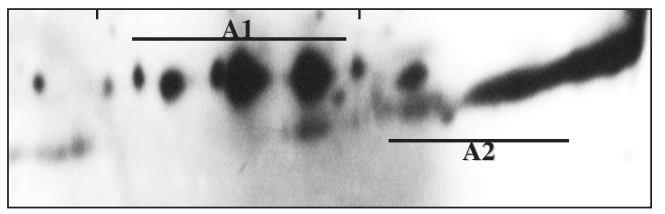

D

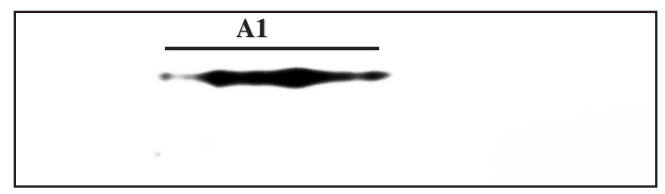

$\mathbf{E}$



Fig. 3. Boxed areas containing annexins I and II and PGP 9.5 proteins shown in Fig. 2. Panel A: Location of PGP 9.5 forms P1, P2 and P3 in a silver stained gel. Panel B: Reactivity of a lung adenocarcinoma patient serum with forms P1-P3. Panel C: Location of annexins I and II in a silver stained gel. Panel D: Reactivity of anti-annexin I monoclonal antibody with form identified as annexin I by mass spectrometry. Panel E: Reactivity of anti-annexin II monoclonal antibody with form identified as annexin II by mass spectrometry.

II, respectively [46,47]. Glycosylation may contribute to protein stability and may enhance signal transduction [44].

Interestingly, IL-6 levels were significantly higher in sera of antibody-positive lung cancer patients compared with antibody-negative patients and controls. This led us to conclude that an immune response manifested by annexins I and II autoantibodies occurs commonly in lung cancer and is associated with high circulating levels of an inflammatory cytokine.

\section{Conclusion}

The initial proteomic approach we have implemented was based on the analysis of whole tissue or whole cell lysates. We have demonstrated that this approach has utility for the identification of differentially expressed proteins and for the development of serum based assays for cancer diagnosis. The approach is currently being expanded in several ways. First, proteomic analysis is increasingly focused on the analysis of individual sub-cellular compartments such as surface membranes, nuclear proteins etc. Second the 2-D gel based approach is increasingly complemented with other separation modes such as the use of multi-dimensional liquid chromatography that is particularly suited for automation and for the analysis of small molecular weight proteins and peptides. Other emerging technologies include the use of protein microarrays in which antigen or antibody, representing probes, is deposited onto glass surfaces and interrogated with targets represented by reagents, sera, biological fluids or cell or tissue lysates. One would envisage the development of specialized microarrays to interrogate targets relevant to lung cancer. Proteomics is likely to contribute substantially to our understanding of the pathophysiology of lung cancer and to the development of novel diagnostics and therapeutics.

\section{References}

[1] A.C. Bergman et al., Identification of gel-separated tumor marker proteins by mass spectrometry, Electrophoresis 21 (2000), 679-686.

[2] T. Hirano et al., Detection of polypeptides associated with the histopathological differentiation of primary lung carcinoma, Br. J. Cancer 72 (1995), 840-848.

[3] H.R. Schmid et al., Lung tumor cells: a multivariate approach to cell classification using two-dimensional protein pattern, Electrophoresis 16 (1995), 1961-1968.

[4] A. Yamamoto et al., Detection of auto-antibodies against Lmyc oncogene products in sera from lung cancer patients, Int J Cancer 22 (1996), 283-289.

[5] E. Stockert et al., A survey of the humoral immune response of cancer patients to a panel of human tumor antigens, J Exp Med 187 (1998), 1349-1354.

[6] A.O. Gure et al., Human lung cancer antigens recognized by autologous antibodies: Definition of a novel cDNA derived from the tumor suppressor gene locus on chromosome $3 \mathrm{p} 21.3$, Cancer Res 58 (1998), 1034-1341.

[7] T. Soussi, p53 Antibodies in the sera of patients with various types of cancer: a review, Cancer Res 60 (2000), 1777-1788.

[8] A. Yamamoto et al., Infrequent presence of anti-c-Myc antibodies and absence of c-Myce oncoprotein in sera from lung cancer patients, Oncology 56(2) (1999), 129-133.

[9] P. Maddison et al., Favourable prognosis in Lambert-Eaton myasthenic syndrome and small-cell lung carcinoma, Lancet 353(9147) (1999), 117-118.

[10] F. Blaes et al., Antineural and antinuclear autoantibodies are of prognostic relevance in non-small cell lung cancer, Ann. Thorac. Surg 69(1) (2000), 254-258.

[11] Y. Hirasawa et al., Natural autoantibody to MUC1 is a prognostic indicator for non-small cell lung cancer, Am J Respir Crit Care Med 161 (2000), 589-594. 
[12] R.B. Darnell and L.M. DeAngelis, Regression of small-cell lung carcinoma in patients with paraneoplastic neuronal antibodies, Lancet 341(8836) (1993), 21-22.

[13] R.B. Darnell, Onconeural antigens and the paraneoplastic neurologic disorders: at the intersection of cancer, immunity, and the brain, Proc. Natl. Acad. Sci. USA 93(10) (1996), 45294536.

[14] H. Yanagawa et al., Serum levels of interleukin 6 in patients with lung cancer, Br J Cancer 71(5) (1995), 1095-1098.

[15] F. Martin et al., Cytokine levels (IL-6 and IFN-gamma), acute phase response and nutritional status as prognostic factors in lung cancer, Cytokine 11(1) (1999), 80-86.

[16] J.R. Strahler, R. Kuick and S.M. Hanash, Two-dimensional polyacrylamide gel electrophoresis of proteins, in: Protein Structure: A Practical Approach, T. Creighton, ed., IRL Press Ltd., Oxford, 1989, pp. 65-92.

[17] F. Gharahdaghi et al., Mass spectrometric identification of proteins from silver-stained polyacrylamide gel: a method for the removal of silver ions to enhance sensitivity, Electrophoresis 20(3) (1999), 601-605.

[18] K.C. Gatter and C.L. Oakley lecture, Diagnostic immunocytochemistry: achievements and challenges, J Pathol 159(3) (1989), 183-190.

[19] C. Asselin-Paturel et al., Quantitative analysis of Th1, Th2 and TGF-beta1 cytokine expression in tumor, TIL and PBL of non-small cell lung cancer patients, Int J Cancer 77(1) (1998), 7-12.

[20] M.M. Gourevitch et al., Polymorphic epithelial mucin (MUC1)-containing circulating immune complexes in carcinoma patients, Br. J. Cancer 72 (1995), 934-938.

[21] T. Soussi, The humoral response to the tumor-suppressor gene product p53 in human cancer: Implications for diagnosis and therapy, Immunol. Today 17 (1996), 354-356.

[22] L.J. Old and Y.T. Chen, New paths in human cancer serology, J Exp Med 187 (1998), 1163-1167.

[23] L. Prasannan et al., Identification of beta-tubulin isoforms as tumor antigens in neuroblastoma, Clin. Cancer Res. 6(10) (2000), 3949-3956.

[24] F. Brichory et al., Proteomics-based identification of PGP 9.5 as a tumor antigen that induces a humoral immune response in lung cancer, Cancer Res., in press.

[25] F.M. Brichory et al., An immune response manifested by common occurrence of annexins I and II autoantibodies and high circulating levels of interleukin 6 in lung cancer, submitted.

[26] I.N. Day and R.J. Thompson, Molecular cloning of cDNA coding for human PGP 9.5 protein. A novel cytoplasmic marker for neurones and neuroendocrine cells, Febs Lett. 210(2) (1987), 157-160.

[27] A. Hershko and A. Ciechanover, The ubiquitin system, Ann. Rev. Biochem. 67 (1998), 425-479.

[28] V. Spataro, C. Norbury and A.L. Harris, The ubiquitinproteasome pathway in cancer, Br. J. Cancer 77(3) (1998), 448-455.

[29] M. Hochstrasser, Ubiquitin-dependent protein degradation, Annu. Rev. Genet. 30 (1996), 405-439.
[30] B.J. Addis et al., Immunohistochemical markers of small cell carcinoma and related neuroendocrine tumours of the lung, $J$. Pathol. 153(2) (1987), 137-150.

[31] K. Hibi et al., Serial analysis of gene expression in non-small cell lung cancer, Cancer Res. 58(24) (1998), 5690-5694.

[32] G. Abbona et al., Chromogranin A gene expression in nonsmall cell lung carcinomas, J. Pathol. 186(2) (1998), 151-156.

[33] A.P. Dhillon et al., Neural markers in carcinoma of the lung, Br. J. Cancer 51(5) (1985), 645-652.

[34] K. Hibi et al., PGP9.5 as a candidate tumor marker for nonsmall-cell lung cancer, Am. J. Pathol. 155(3) (1999), 711-715.

[35] J. Mai et al., Human procathepsin B interacts with annexin II tetramer on the surface of tumor cells, J Biol Chem 275(17) (2000), 12806-12812.

[36] W. Pruzanski et al., Circulating group II phospholipase A2 activity and antilipocortin antibodies in systemic lupus erythematosus. Correlative study with disease activity, J. Rheumatol. 21(2) (1994), 252-257.

[37] M.R. Podgorski et al., Autoantibodies to lipocortin-1 are associated with impaired glucocorticoid responsiveness in rheumatoid arthritis, J. Rheumatol. 19(11) (1992), 1668-1671.

[38] F. Goulet, K.G. Moore and A.C. Sartorelli, Glycosylation of annexin I and annexin II, Biochem. Biophys. Res. Commun. 188(2) (1992), 554-558.

[39] T. Dubois et al., Annexins and protein kinases C, Biochim. Biophys. Acta 1313(3) (1996), 290-294.

[40] B.P. Wallner et al., Cloning and expression of human lipocortin, a phospholipase A2 inhibitor with potential antiinflammatory activity, Nature 320(6057) (1986), 77-81.

[41] T. Kristensen et al., Primary structure of bovine calpactin I heavy chain (p36), a major cellular substrate for retroviral protein-tyrosine kinases: homology with the human phospholipase A2 inhibitor lipocortin, Biochem 25(16) (1986), $4497-$ 4503.

[42] A. Gure et al., Serological identification of embryonic neural proteins as highly immunogenic tumor antigens in small cell lung cancer, Proc. Natl. Acad. Sci. USA 97(8) (2000), 41984203.

[43] A. Dowlati, N. Levitan and S.C. Remick, Evaluation of interleukin-6 in bronchoalveolar lavage fluid and serum of patients with lung cancer, J Lab Clin Med 134(4) (1999), 405409.

[44] E. Solito et al., IL-6 stimulates annexin 1 expression and translocation and suggests a new biological role as class II acute phase protein, Cytokine 10(7) (1998), 514-521.

[45] K. Li et al., Tumour-specific MHC-class-II-restricted responses after in vitro sensitization to synthetic peptides corresponding to gp100 and Annexin II eluted from melanoma cells, Cancer Immunol Immunother 47(1) (1998), 32-38.

[46] U. Boehm et al., Cellular responses to interferon-gamma, Ann. Rev. Immunol. 15 (1997), 749-795.

[47] M.D. Johnson et al., Lipocortin-1 immunoreactivity in central and peripheral nervous system glial tumors, Hum. Pathol. 20(8) (1989), 772-776. 




The Scientific World Journal




Gastroenterology

Research and Practice
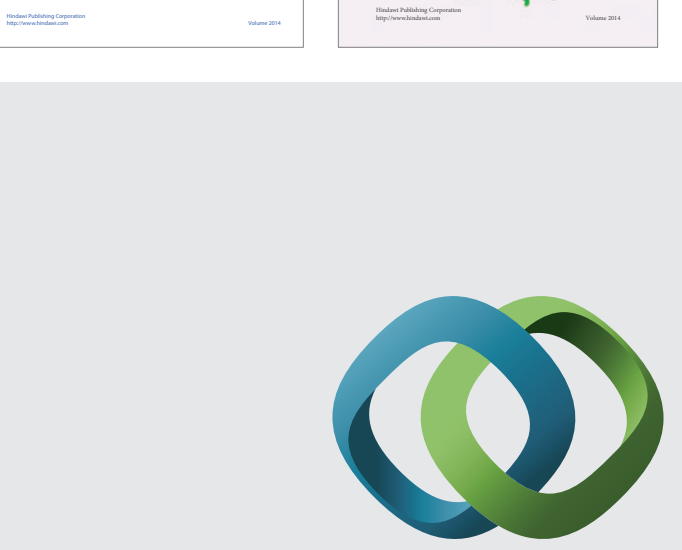

\section{Hindawi}

Submit your manuscripts at

http://www.hindawi.com
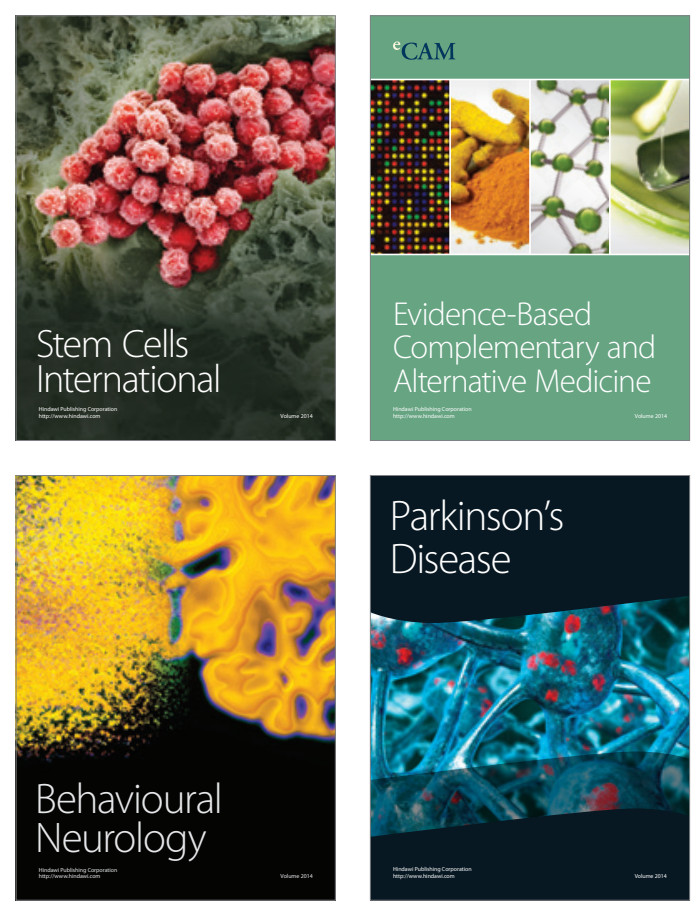



Journal of
Diabetes Research

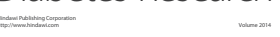



Disease Markers
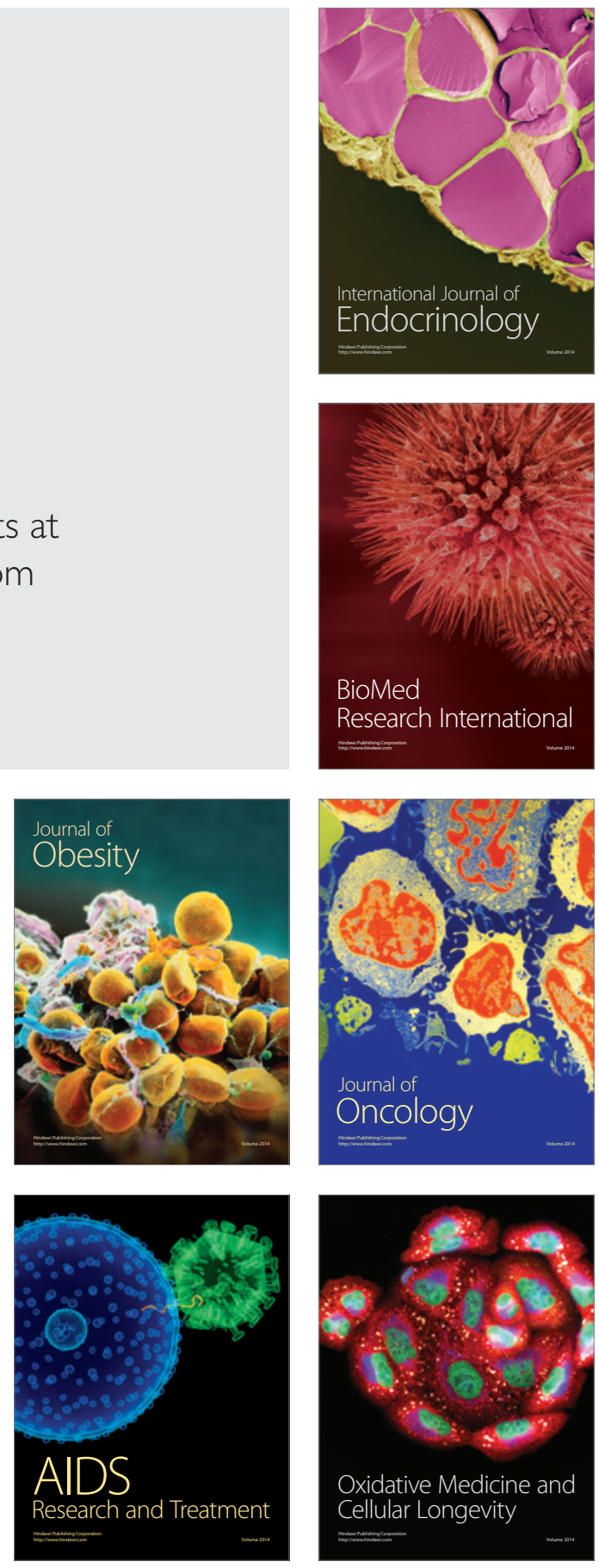